\title{
Volitional electromyographic responses in disorders of consciousness
}

\author{
Dina Habbal ${ }^{1}$, Olivia Gosseries ${ }^{1}$, Quentin Noirhomme ${ }^{1}$, Jerome Renaux ${ }^{1}$, Damien Lesenfants ${ }^{1}$, \\ Tristan A. Bekinschtein ${ }^{2}$, Steve Majerus ${ }^{3}$, Steven Laureys ${ }^{1}$, \& Caroline Schnakers $^{1,3,4}$ \\ ${ }^{1}$ Coma Science Group, Cyclotron Research Center and Neurology Department, University and University Hospital of Liège, Liège, Belgium, \\ ${ }^{2}$ Cognition and Brain Sciences Unit, Medical Research Council, Cambridge, UK, ${ }^{3}$ Department of Psychology, Cognition and Behaviour, \\ University of Liège, Liège, Belgium, and ${ }^{4}$ Department of Psychology and Department of Neurosurgery, University of California, Los Angeles, CA, USA
}

\section{Abstract}

Objective: The aim of the study was to validate the use of electromyography (EMG) for detecting responses to command in patients in vegetative state/unresponsive wakefulness syndrome (VS/UWS) or in minimally conscious state (MCS).

Methods: Thirty-eight patients were included in the study (23 traumatic, 25 patients $>1$ year post-onset), 10 diagnosed as being in VS/UWS, eight in MCS- (no response to command) and 20 in MCS+ (response to command). Eighteen age-matched controls participated in the experiment. The paradigm consisted of three commands (i.e. 'Move your hands', 'Move your legs' and 'Clench your teeth') and one control sentence (i.e. 'It is a sunny day') presented in random order. Each auditory stimulus was repeated 4-times within one block with a stimulus-onset asynchrony of 30 seconds.

Results: Post-hoc analyses with Bonferroni correction revealed that EMG activity was higher solely for the target command in one patient in permanent VS/UWS and in three patients in MCS+.

Conclusion: The use of EMG could help clinicians to detect conscious patients who do not show any volitional response during standard behavioural assessments. However, further investigations should determine the sensitivity of EMG as compared to neuroimaging and electrophysiological assessments.
\end{abstract}

\section{Introduction}

In order to diagnose a patient recovering from coma as being conscious [1], clinicians search for reproducible behavioural responses which are considered as localizing or, better, volitional. Contrary to localizing responses (e.g. oriented eye movements; see the debate around visual fixation) [2], the conscious aspect of volitional responses is difficult to dispute (a patient has to be conscious in order to wilfully respond to stimulation). These responses are, therefore, crucial to detect. The most common volitional behaviour in this field is the response to simple commands (i.e. the ability to execute a simple movement on demand). Besides its volitional aspect, the response to command is assumed to indicate the reappearance of higher-level cognitive functions such as language (i.e. comprehension of the command) and executive functions (i.e. accurate planning of the movement execution). The recovery of these functions may form the bases for communication between the patient and the medical staff and

Correspondence: Caroline Schnakers, Department of Psychology and Neurosurgery, University of California, Los Angeles, CA 90095-1563. Tel: +1 310-825-8546. E-mail: cschnakers@ucla.edu

\section{Keywords}

Brain injury, consciousness, electromyogram, minimally conscious state, unresponsive wakefulness syndrome, vegetative state

History

Received 2 July 2013

Revised 6 September 2013

Accepted 25 October 2013

Published online 6 June 2014 for a more active role of the patient in his/her own rehabilitation. The detection of responses to command is, nevertheless, complicated by the limited motor responses and the fatigability of patients with disorders of consciousness (DOC). Recent studies have shown how difficult it is, even for trained clinicians, to differentiate volitional from reflexive behaviours, leading to high misdiagnosis of conscious patients (rate of $\sim 40 \%$ ) [3-5]. In fact, even when using sensitive standardized diagnostic scale such as the Coma Recovery Scale-Revised (CRS-R) [6], micromovements might still remain undetected at the patient's bedside. Recently, Bekinschtein et al. [7] used electromyography (EMG) to improve the detection of responses to command. In several patients with no reproducible volitional responses at the bedside (including one patient in minimally conscious state-MCS but also one patient diagnosed as being in a vegetative state-VS or, more recently, unresponsive wakefulness syndrome - UWS) [8, 9], the authors found a significant increase of the EMG signal in response to a target command (i.e. 'Move your hand') as opposed to a control command (e.g. 'Today is a sunny day'). This technique may constitute an objective measure to better detect volitional behaviours in patients with DOC. It would also represent a more convenient tool as compared to neuroimaging or electrophysiology as it is portable, easy to use and 
inexpensive. However, Bekinschtein et al.'s [7] study only included 10 patients (i.e. eight VS/UWS and two MCS), making clinical inferences very difficult. The aim of this study is, therefore, to test this technique in a larger sample, taking into account three different commands in order to confirm the interest of electromyography (EMG) for the detection of volitional responses in patients with severe brain injury.

\section{Methods}

\section{Inclusion/exclusion criteria}

Patients were recruited from the University Hospital of Liège (CHU-Liège, Belgium). Medically stable patients were assessed with the CRS-R and diagnosed as being in VS/UWS or MCS (traumatic and non-traumatic causes). Inclusion criteria were (a) age above 18, (b) at least 28 days post-injury, (c) preserved auditory evoked potentials and/or presence of auditory startle and (d) no neuromuscular function blockers and no sedation within the prior 24 hours. Exclusion criteria were (a) documented history of prior brain injury, (b) pre-morbid history of developmental, psychiatric or neurologic illness resulting in documented functional disability up to the time of the injury, (c) pre-morbid history of uncorrected hearing impairments, (d) flaccidity in response to noxious stimulation and (e) acute illness. The patients' group was age-matched to healthy volunteers (control group). For this group, exclusion criteria were (a) uncorrected hearing impairments, (b) muscle disease or muscle dysfunction due to an injury and (c) developmental, psychiatric or neurologic illness. The study was approved by the ethics committee of the Faculty of Medicine, University of Liège. Written informed consent was obtained from the patients' legal surrogate.

\section{Behavioural assessment}

The CRS-R was administered on the day of the EMG recording as well as several times during the week of the recording to establish the participant's diagnosis and neurobehavioural profile. This scale was designed to differentiate VS/UWS from MCS and consists of 23 hierarchicallyarranged items that comprise six sub-scales assessing arousal, auditory, visual, motor, oromotor/verbal and communication functions. The lowest item on each sub-scale represents reflexive activity, while the highest item represents cognitively-mediated behaviours [6]. As behavioural fluctuation is frequent in patients with severe brain injury [10], the highest CRS-R total score obtained was reported. Based on these behavioural assessments, the sample was divided into the following sub-groups: MCS+ [11] (if any reproducible response to command was obtained at the bedside; CRS-R Auditory sub-score >2), MCS - [11] (if no reproducible response to command but only non-reflexive behaviours such as visual pursuit or localization to noxious stimulation were obtained at the bedside; CRS-R Auditory sub-score $\leq 2$ ) and VS/UWS (if no localizing or volitional response was obtained at the bedside; CRS-R sub-scores as follows: Auditory $\leq 2$; Visual $<2$; Motor $<3$; Oromotor $<3$; Communication $<1$; Arousal $<3$ ).

\section{EMG paradigm}

Pre-recorded auditory stimuli were presented in a random order $(90 \mathrm{~dB})$. The stimuli consisted of three target commands (i.e. 'Move your hands', 'Move your legs' and 'Clench your teeth') as well as one control auditory phrase (i.e. 'It is a sunny day'). All stimuli, although different in semantic information, had the same length (1.8 seconds) and approximately the same intonation. In order to follow the clinical guidelines of the standardized behavioural tool [6], each auditory stimulus was repeated 4-times within one block. The stimulus onset asynchrony was of 30 seconds. The total duration of the task was less than 10 minutes.

\section{EMG recording}

Surface electromyogram (EMG) activity was recorded at the patients' bedside from eight disposable adhesive surface electrodes (www.spesmedica.com) which were applied using a bipolar montage (inter electrode distance of $20 \mathrm{~mm}$ ) and connected to a portable digital EEG/EMG amplifier (SynAmp EEG amplifier; www.neuroscan.com). The electrodes were placed on the abductor policis brevis muscle $(n=2$; channel 'Hand') and on the flexor digitorum superficialis muscle $(n=2$; channel 'Arm') for recording responses to the command 'Move your hands', on the gastrocnemius muscle ( $n=2$; channel 'Leg') for the command 'Move your legs' (www.seniam.org) as well as on the masseter muscle $(n=2$; channel 'Face') for the command 'Clench your teeth' $[12,13]$. The electrodes were placed on the side (i.e. left or right) of the upper and lower limbs that presented the best motor responses during behavioural assessments. Data were recorded on a laptop computer. EMG acquisition was performed with a sampling rate of $2000 \mathrm{~Hz}$ and a band pass filtering of $0.1-500 \mathrm{~Hz}$ was used [14]. This procedure lasted $\sim 15$ minutes. Before starting the recording, upper and lower limbs as well as jaw were passively moved in order to assure the accuracy of the electrodes position. EMG recordings were performed while the patients were in a wakeful state with eyes open in a setting with minimal ambient noise. Brief auditory or deep pressure stimulation, as used by the CRS-R [6], was applied immediately before presenting each command in order to ensure sufficient arousal. During the recording, any response observed behaviourally was reported. Note that two different muscle groups were tested for the command 'Move your hands' to ensure that the initial method (using abductor policis brevis muscle) was the most efficient one to detect responses.

\section{EMG analyses}

The EMG signal was filtered with a Butterworth filter between $20-400 \mathrm{~Hz}$ [14]. For each participant, the average value of the EMG amplitude (in $\mu \mathrm{v}$ ) per second was calculated at each channel (i.e. 'Hand', 'Arm', 'Leg' and 'Face') for each command on each trial $(n=4)$. EMG data (absolute values) obtained at the channel matching one of the commands (e.g. channel 'Leg' for the command 'Move your legs') were analysed using an ANOVA with repeated measures on the type of command (target vs control command) and the number of trials (1-4). To ensure that 
responses were reproducible and reliable, this study followed the clinical guidelines of the standardized behavioural tool [6] and considered a response to command as present in a patient if the EMG activity was higher for the target command than for the control command in at least three out of four trials, using post-hoc analyses with Bonferroni correction. Results were thresholded at $p$ value $\leq 0.05$.

\section{Results}

\section{Participants}

Thirty-eight patients with severe brain injury $(39 \pm 14$ years old; 20 men) were included in this study. The aetiology of brain injury was traumatic $(n=23)$, anoxic $(n=5)$, haemorrhagic $(n=4)$, stroke $(n=2)$, encephalitis $(n=1)$ or mixed (i.e. traumatic and anoxic; $n=3$ ). Twenty-five patients had been in a DOC for more than a year (from 1.08-11.83 years post-insult) and 13 for less than a year (from 51-347 days post-insult). Ten patients were diagnosed as being in VS/ UWS, eight in MCS - and 20 in MCS+ according to CRS-R scores (see Table I). Two patients demonstrated either functional communication or functional object use during one assessment, but this performance was not observed consistently over two consecutive assessments and, hence, did not correspond to the criteria for emergence from MCS [1]. Eighteen controls ( $32 \pm 12$ years old; five men) also participated in the experiment.

\section{EMG results}

For the controls (see Figure 1), EMG activity was significantly higher as compared to the control command in $83 \%$ of cases (i.e. 15/18) for the command 'Move your hands' (at the channel 'Arm') and for the command 'Clench your teeth', in $67 \%$ of cases (i.e. 12/18) for the command 'Move your hands' (at the channel 'Hand') and in 61\% of cases (i.e. 11/18) for the command 'Move your legs'. For the patients, a response to command was obtained for at least one of the target commands in six VS/UWS, three MCS - and 11 MCS+. Since these patients often suffer severe spasticity and may present involuntary/hypertonic muscle spasms [15-17], additional ANOVAs were performed in order to ensure that the increase of EMG activity was significantly higher for the target command as compared to the two other non-target commands (e.g. higher EMG activity at the channel 'Leg' for the command 'Move your legs' as compared to the commands 'Move your hands' and 'Clench your teeth'). This had to be true solely for the channel matching the target command (e.g. higher EMG activity at the channel 'Leg' for the command 'Move your legs' and not at channels 'Hand', 'Arm' or 'Face'). Post-hoc analyses with Bonferroni correction revealed that EMG activity was significantly higher solely for the target command in one VS/UWS (i.e. VS/UWS 5) and three MCS+ (i.e. MCS+ 1, MCS+ 2 and MCS+ 6). Three patients responded to 'Move your hands' and one patient responded to 'Move your legs' (see Table II and Figures 2 and 3). During CRS-R assessments, reproducible responses to command were obtained in MCS +1 and MCS +6 using 'move your hand' and in MCS+ 2 using 'move your hand' but also 'move your head'. During the EMG recording, responses to command were detectable behaviourally (by the naked eye) for at least three out of four trials in MCS+ 1 and MCS+ 2, but for none of the trials in VS/UWS 5 and MCS+ 6 .

\section{Discussion}

The aim of this study was to confirm the interest of electromyography (EMG) for the detection of responses to command in patients with a severe brain injury. As in the original study by Bekinschtein et al. [7], a significant increase of EMG signal was elicited to command in a patient who did not show any volitional behavioural response and was diagnosed as being in permanent VS/UWS (i.e. time postanoxia longer than 3 months-here, 4.59 years).

Volitional brain activity has previously been detected in patients considered as being in VS/UWS using neuroimaging $[18,19]$ and electrophysiology [20, 21]. Those studies have concluded that high-level cognition can be observed in the absence of purposeful motor responses, suggesting a potential dissociation between behavioural expression of consciousness (involving motor functions) and consciousness per se (involving cognitive functions) [22]. None of those studies, nevertheless, checked for the presence of volitional micromovements. Only one fMRI study found, in two out of five patients in VS/UWS, functional changes in the premotor cortex elicited by specific verbal commands (i.e. move your left/right hand), whereas no volitional response was found using EMG recordings [23]. EMG and fMRI recordings were, however, performed separately in this study, making it impossible to reject the intervention of vigilance fluctuations as a potential bias. As patients with severe brain injury (particularly those in a chronic stage) often present motor deficits due to severe spasticity [16, 17], it is likely that volitional movements will not be visually detectable at the bedside. The results suggest that future studies should perform simultaneous EMG and neuroimaging/electroencephalographical recordings in order to better understand the interaction between motor and cognitive functions in those patients. Investigating whether EMG has equal or higher sensitivity to detect volitional responses over neuroimaging and other electrophysiological techniques also presents a real clinical interest, as EMG recordings are inexpensive and can be conducted rapidly. In order to ensure an optimal rate of response, the command 'Move your hands' should be selected. According to these results, this command was the one for which responses were the most frequently observed in both controls (83\% of cases) and patients (three out of four responsive patients). Responses differed according to electrodes position. Indeed, a higher rate of responses was detected at the channel 'Arm' (i.e. digitorum superficialis muscle) vs 'Hand' (i.e. abductor policis brevis muscle) in controls ( $83 \%$ vs $67 \%$ of cases, respectively), whereas responses were observed at both channels in patients, suggesting that the assessment of both muscle groups should be used in future investigations.

As hypertonic muscle spasms are frequent in patients with severe brain injury [15], involuntary movements could be mistakenly considered as a volitional response leading to false positive results. For this reason, a response was considered as present in this study if EMG activity was significantly higher 


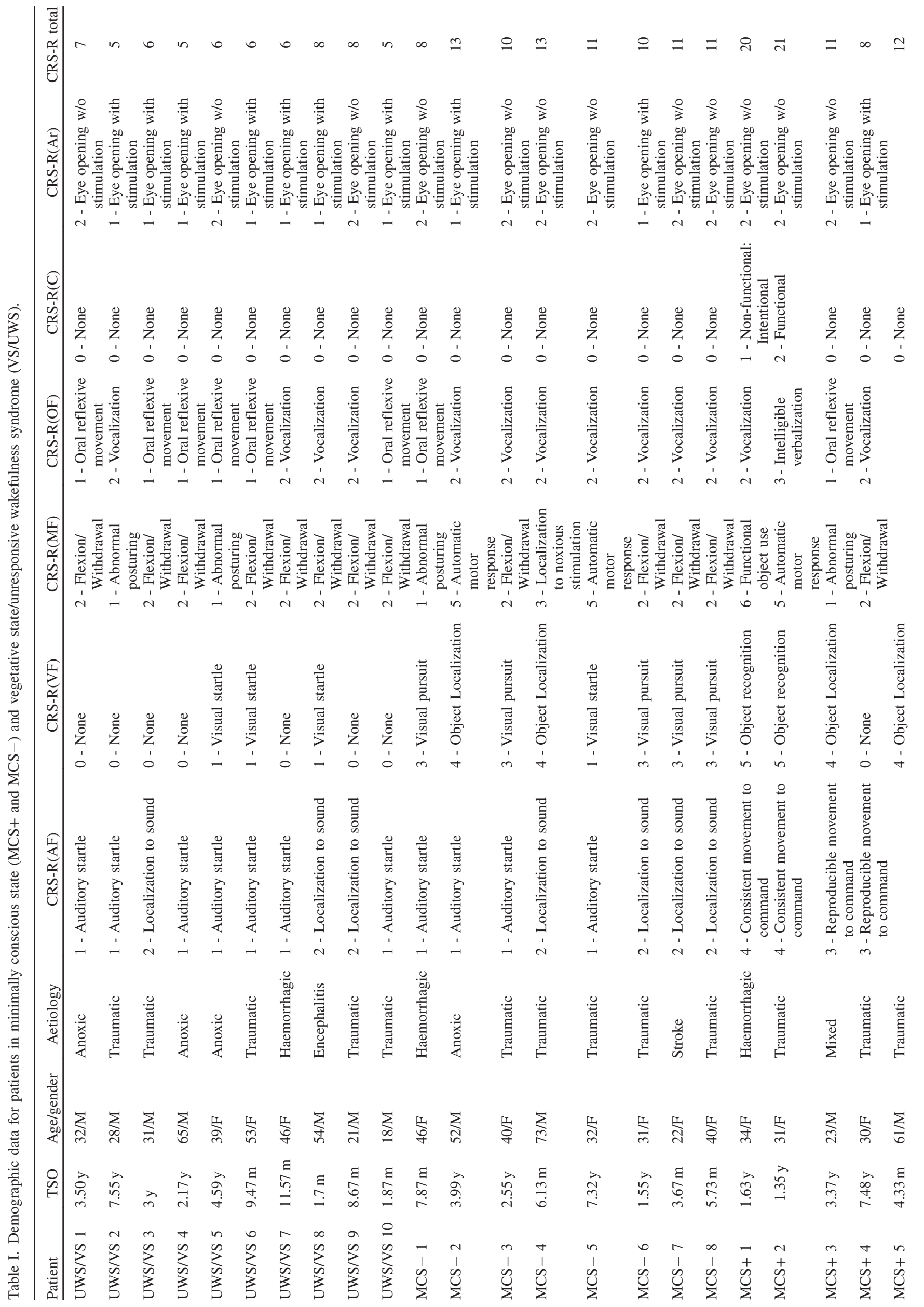




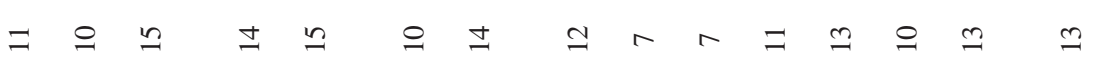

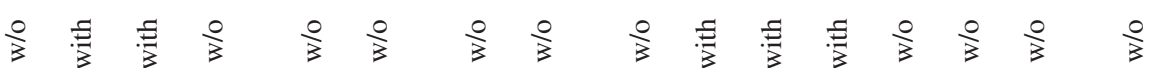

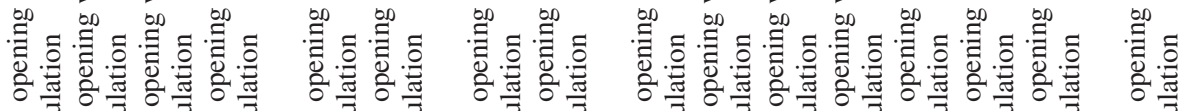

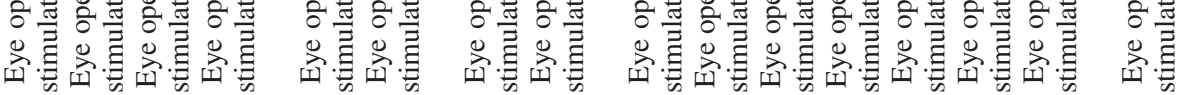

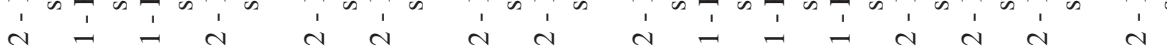

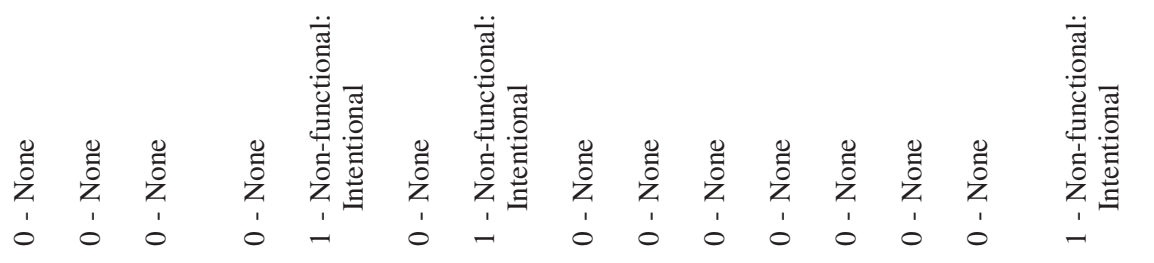

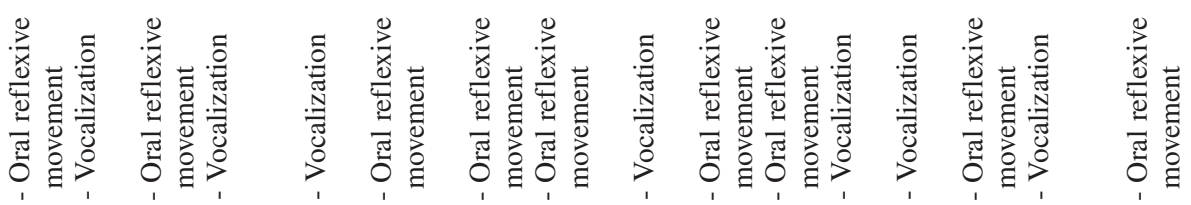

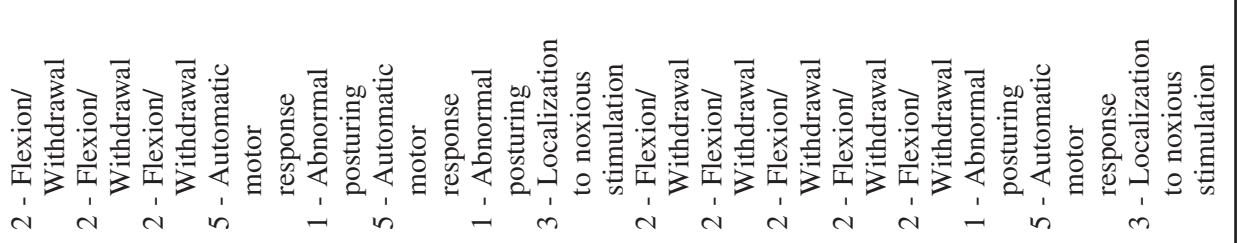

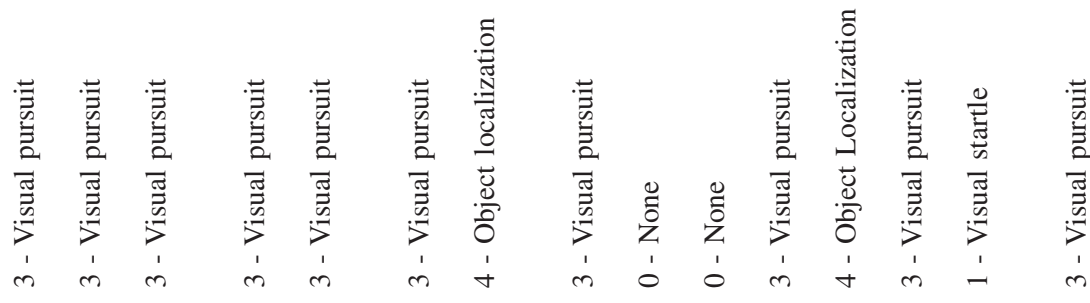

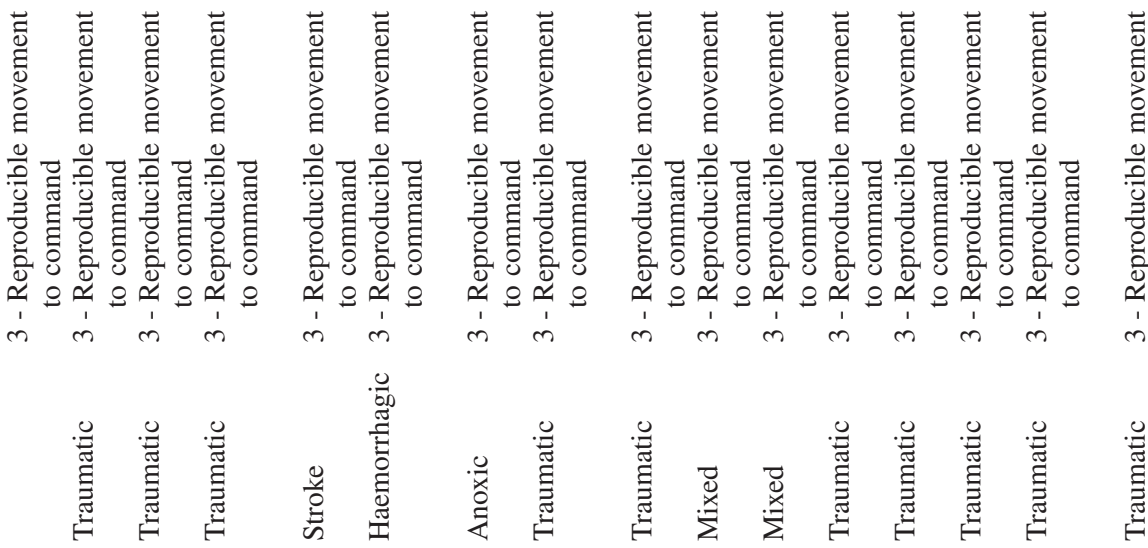

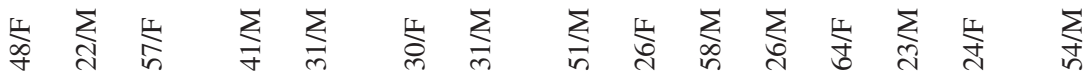

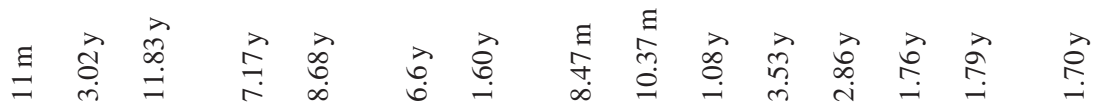

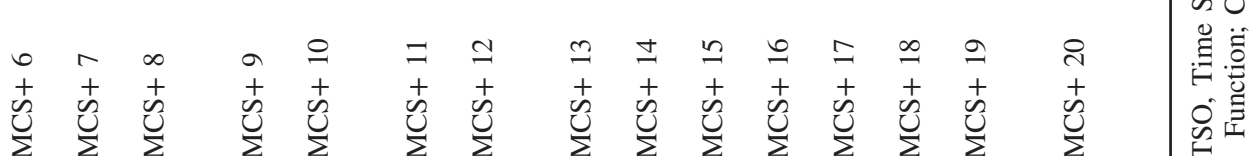



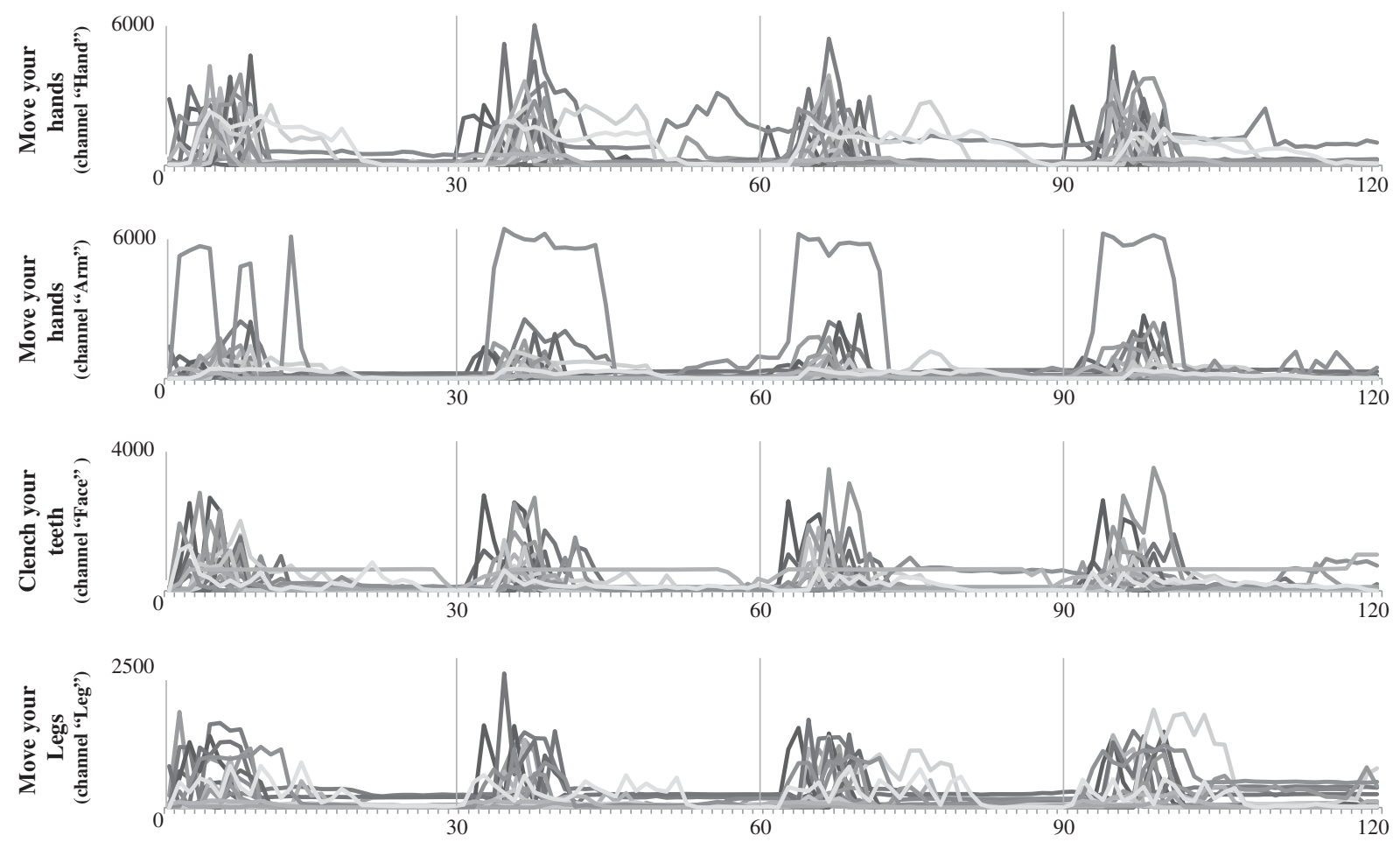

Figure 1. EMG activity in controls $(n=18)$. This figure illustrates the amplitude (in $\mu \mathrm{v} ; y$-axes) of the EMG activity across trials $(n=4)$ at the channel matching the target command (e.g. EMG activity at the channel 'Hand' for the command 'Move your hands') for each of the 18 controls (in various grey). The $x$-axes represent time in seconds.

Table II. Number of trials (maximum of four per command) for which a higher EMG activity was observed in patients in MCS+, MCS - and VS/UWS.

\begin{tabular}{|c|c|c|c|c|}
\hline$\underline{\text { Patients }}$ & $\begin{array}{l}\text { Move your } \\
\text { hands } \\
\text { (channel } \\
\text { 'Hand') } \\
\end{array}$ & $\begin{array}{l}\text { Move your } \\
\text { hands } \\
\text { (channel } \\
\text { 'Arm') } \\
\end{array}$ & $\begin{array}{c}\text { Clench your } \\
\text { teeth } \\
\text { (channel } \\
\text { 'Face') } \\
\end{array}$ & $\begin{array}{c}\text { Move your } \\
\text { legs } \\
\text { (channel } \\
\text { 'Leg') } \\
\end{array}$ \\
\hline UWS/VS 1 & 0 & 1 & 0 & 1 \\
\hline UWS/VS 2 & $4 *$ & 0 & 0 & 2 \\
\hline UWS/VS 3 & 0 & 2 & 1 & 0 \\
\hline UWS/VS 4 & 1 & 0 & 0 & 1 \\
\hline UWS/VS 5 & $3 * *$ & 0 & 0 & 0 \\
\hline UWS/VS 6 & $4 *$ & $3 *$ & $4 *$ & 0 \\
\hline UWS/VS 7 & 1 & $4^{*}$ & 0 & 1 \\
\hline UWS/VS 8 & 1 & 1 & 0 & $3 *$ \\
\hline UWS/VS 9 & 0 & $3^{*}$ & 2 & 0 \\
\hline UWS/VS 10 & 1 & 0 & 0 & 2 \\
\hline $\mathrm{MCS}-1$ & 2 & 2 & $3 *$ & $3 *$ \\
\hline $\mathrm{MCS}-2$ & 0 & 2 & 2 & 1 \\
\hline MCS -3 & 0 & 0 & 1 & 2 \\
\hline $\mathrm{MCS}-4$ & 0 & 0 & 2 & 1 \\
\hline $\mathrm{MCS}-5$ & 1 & 0 & 1 & 0 \\
\hline MCS -6 & 0 & 0 & 1 & $3 *$ \\
\hline MCS- 7 & 0 & 0 & 0 & 1 \\
\hline MCS -8 & 1 & 2 & $4^{*}$ & 0 \\
\hline $\mathrm{MCS}+1$ & $4 * *$ & $4 * *$ & 2 & 0 \\
\hline $\mathrm{MCS}+2$ & 1 & 0 & 0 & $3 * *$ \\
\hline $\mathrm{MCS}+3$ & $4^{*}$ & 1 & 2 & 0 \\
\hline $\mathrm{MCS}+4$ & $3 *$ & 2 & 1 & 2 \\
\hline $\mathrm{MCS}+5$ & 1 & $4 *$ & $4 *$ & 0 \\
\hline $\mathrm{MCS}+6$ & $4 * *$ & 1 & $3 *$ & 0 \\
\hline $\mathrm{MCS}+7$ & 0 & 0 & 0 & 0 \\
\hline $\mathrm{MCS}+8$ & $4 *$ & 1 & 0 & 1 \\
\hline $\mathrm{MCS}+9$ & 0 & 0 & 1 & 2 \\
\hline $\mathrm{MCS}+10$ & 1 & 0 & 1 & 2 \\
\hline
\end{tabular}

(continued)
Table II. Continued

\begin{tabular}{|c|c|c|c|c|}
\hline Patients & $\begin{array}{l}\text { Move your } \\
\text { hands } \\
\text { (channel } \\
\text { 'Hand') }\end{array}$ & $\begin{array}{l}\text { Move your } \\
\text { hands } \\
\text { (channel } \\
\text { 'Arm') }\end{array}$ & $\begin{array}{c}\text { Clench your } \\
\text { teeth } \\
\text { (channel } \\
\text { 'Face') } \\
\end{array}$ & $\begin{array}{c}\text { Move your } \\
\text { legs } \\
\text { (channel } \\
\text { 'Leg') } \\
\end{array}$ \\
\hline MCS+ 11 & 1 & 0 & 1 & 2 \\
\hline $\mathrm{MCS}+12$ & 0 & 0 & 0 & 1 \\
\hline $\mathrm{MCS}+13$ & $4^{*}$ & $3 *$ & 1 & $3 *$ \\
\hline $\mathrm{MCS}+14$ & 0 & 0 & 2 & 0 \\
\hline MCS +15 & 0 & 1 & 0 & 0 \\
\hline $\mathrm{MCS}+16$ & 0 & 0 & $3 *$ & 1 \\
\hline MCS+ 17 & $4 *$ & 0 & 2 & 0 \\
\hline $\mathrm{MCS}+18$ & 2 & 1 & 0 & 0 \\
\hline MCS+ 19 & 0 & $3 *$ & 0 & 0 \\
\hline $\mathrm{MCS}+20$ & 0 & 0 & 0 & 2 \\
\hline
\end{tabular}

*Significantly higher as compared to the control command (post-hoc analyses with Bonferroni correction; $p \leq 0.05$ ); ** means significantly higher as compared to the control command and the two other nontarget commands solely at the channel matching the command (posthoc analyses with Bonferroni correction; $p \leq 0.05$ ).

on several trials (i.e. three out of four trials; suggesting reproducible response) [6] and if it was observed solely for the target command at the matched channel. Considering these criteria, the detection of potential false positive results was noted in 16 patients (i.e. five VS/UWS, three MCS - and eight MCS+) for whom higher EMG activity was observed for unrelated commands at unrelated channels. Since a response to command was observed with the EMG recordings in only three out of $20 \mathrm{MCS}+$ patients, one may consider that it shows the higher sensitivity of behavioural assessment techniques. However, it is important to mention that the EMG 

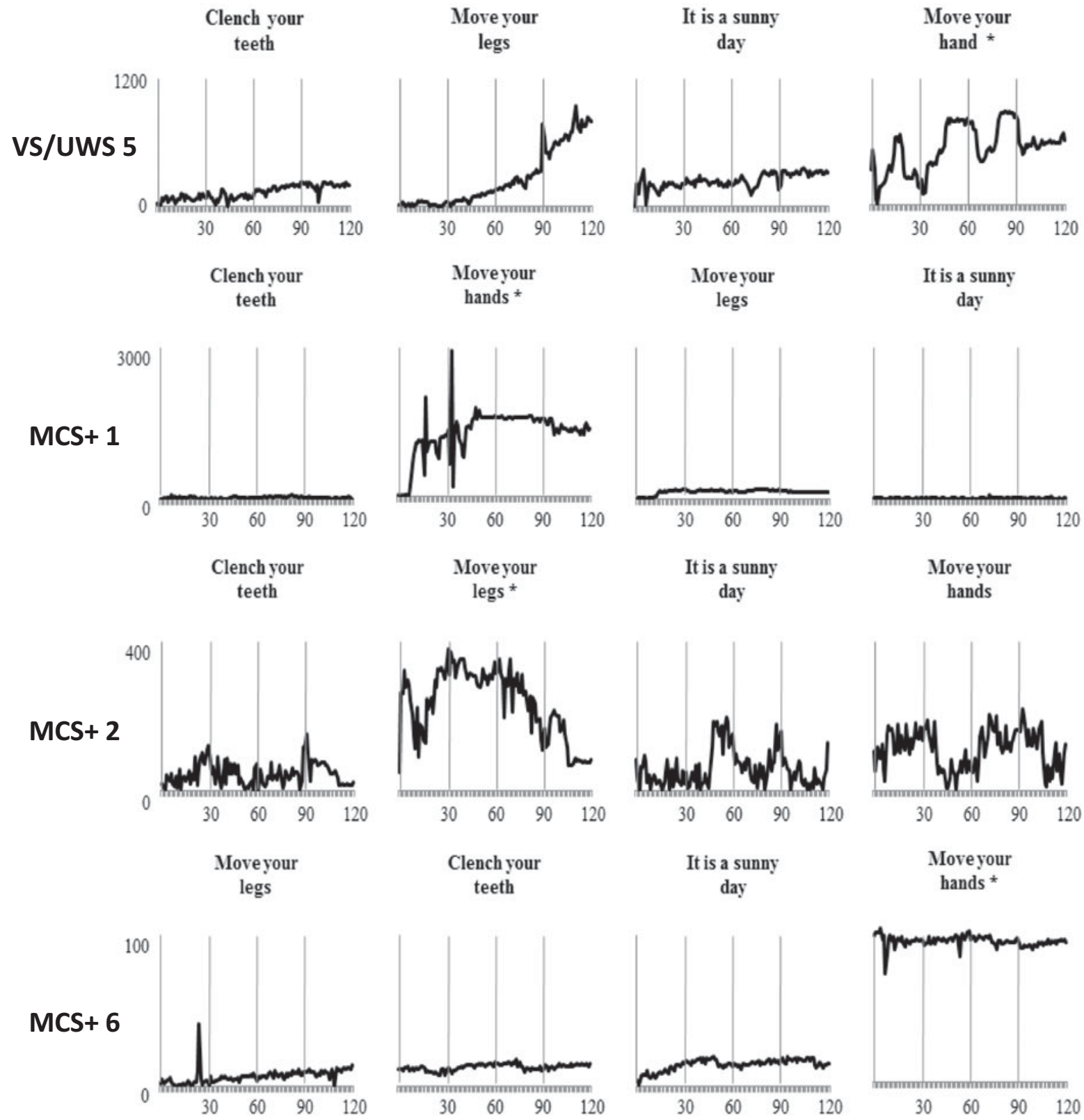

Figure 2. Patients who responded to command through EMG $(n=4)$. This figure illustrates the amplitude (in $\mu$; $y$-axes) of the EMG activity across trials $(n=4)$ at the channel matching the command for which a response was observed (*) (e.g. EMG activity at the channel 'Leg' in MCS+ 6). The order in which the commands are mentioned corresponds to the order of administration during the experiment. The $x$-axes represent time in seconds.

responses acquired in a single session have been compared to a diagnosis based on repeated behavioural assessments. The absence of EMG responses during this unique recording could have been due to vigilance fluctuation, which is a characteristic of the MCS [1]. In fact, in some cases, voluntary muscle responses were observed in the absence of behavioural responses during the EMG recording. In the case of MCS+ 6 , even though he was able to respond during CRS-R assessments, no behavioural response was observed during the EMG recording. One could argue that this is related to performance fluctuations. Voluntary muscle responses were, nevertheless, detected, even though it was not clear behaviourally. Moreover, the fact that a response was observed in a patient diagnosed as being in VS/UWS based on repeated behavioural assessments underlines the interest of EMG, particularly when paramedical data are not characteristic of a VS/UWS. It is indeed worth it to mention that the clinical neuroimaging data of VS/UWS 5 reveal typical brainstem lesions and severe bilateral fronto-parietal atrophy (using structural magnetic resonance imaging-MRI), but also atypically preserved white matter tracts and connectivity within the default mode network (using both diffusion tensor imaging and functional MRI). The latter result being more typical of a MCS [24], the neuroimaging pattern presented by VS/UWS 5 is, therefore, unusual and not characteristic of an unconscious state, stressing further the interest of EMG in helping determining the consciousness level of behaviourally non-responsive patients. Reliable EMG responses were not found in patients in MCS-, which might be explained by previous findings reporting a hypometabolism in the language 


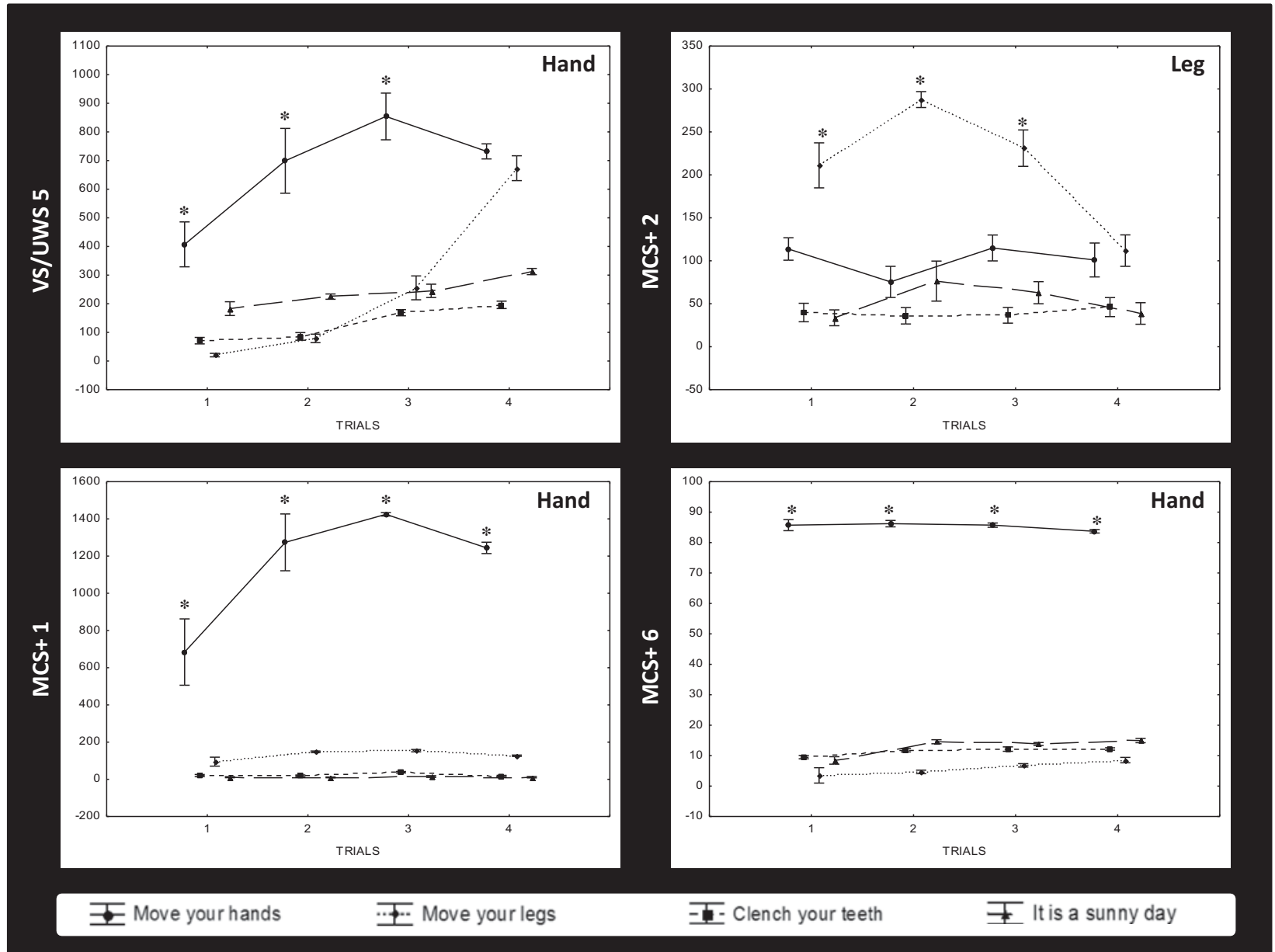

Figure 3. Statistical results in patients who responded to command through EMG $(n=4)$. This figure illustrates the average (in $\mu$ v; $y$-axes) of the EMG amplitude (vertical bars denote 0.95 confidence intervals) across trials $(n=4 ; x$-axes) in response to all commands at the channel matching the command for which a response was observed; *Means significantly higher for the target command as compared to non-target and control commands (post-hoc analyses with Bonferroni correction; $p \leq 0.05$ ).

network in MCS - vs MCS+ and suggesting a deficit in language comprehension [11]. It is, therefore, possible that the patients in MCS - may not have understood the command. On the contrary, a reliable response was found in the only two patients in a MCS+ who were about to emerge from MCS (in fact, MCS+ 2 emerged afterwards) which represents an additional argument in favour of the sensitivity of this technique. Nevertheless, one could argue that this method does not have a good sensitivity as all the controls did not show a higher EMG response for the target command. It is thought that this it is rather linked to the statistical analyses performed. Indeed, the control group had a shorter and sharper response than the patients' group. As the average of the EMG amplitude over 30 seconds (per trial) was compared and not the EMG amplitude around the peak of the response, this might have reduced the average value and might explain why a significant difference was not found for the target vs control command in each healthy volunteer. Future studies should, hence, develop statistics based on peak detection.

In conclusion, the use of EMG for detecting responses to command in patients with severe brain injury could help clinicians in detecting consciousness in patients who do not show any volitional response during behavioural assessments. It is, moreover, an objective technique which is easier to use and less expensive than neuroimaging or electrophysiology. This study detected a volitional response in a patient diagnosed as being in permanent VS/UWS who was able to reliably increase the muscle tone of a target part of her body only when she was asked to do so. Detecting consciousness in patients behaviourally considered as being in VS/UWS is particularly crucial as it has an impact on end-of-life decisions (i.e. the family and the medical staff might change their decision based on the presence of conscious activity). The detection of volitional EMG responses could also be used to allow patients to communicate using residual muscle activity [25]. This would radically improve their autonomy and their quality-of-life. Nevertheless, future studies will have first to determine the sensitivity of EMG as compared to neuroimaging and electrophysiological assessments for detecting consciousness in patients with severe brain injury.

\section{Declaration of interest}

This study was funded by the Belgian National Funds for Scientific Research (FNRS), Fonds Léon Fredericq, James S. McDonnell Foundation; Mind Science Foundation; European Commission; Concerted Research Action; Public Utility Foundation 'Université Européenne du Travail' and 
'Fondazione Europea di Ricerca Biomedica'. This project has also been funded with the support of the European Commission (FP7 DECODER). This publication only reflects the view of the authors and the Commission cannot be held responsible for any use which may be made of the information contained therein. The authors report no conflicts of interest.

\section{References}

1. Giacino JT, Ashwal S, Childs N, Cranford R, Jennett B, Katz DI, Kelly JP, Rosenberg JH, Whyte J, Zafonte RD, et al. The minimally conscious state: Definition and diagnostic criteria. Neurology 2002; 58:349-353.

2. Bruno MA, Vanhaudenhuyse A, Schnakers C, Boly M, Gosseries O, Demertzi A, Majerus S, Moonen G, Hustinx R, Laureys S. Visual fixation in the vegetative state: An observational case series PET study. BMC Neurology 2010;10:35.

3. Schnakers C, Vanhaudenhuyse A, Giacino J, Ventura M, Boly M, Majerus S, Moonen G, Laureys S. Diagnostic accuracy of the vegetative and minimally conscious state: Clinical consensus versus standardized neurobehavioural assessment. BMC Neurology 2009; $9: 35$

4. Andrews K, Murphy L, Munday R, Littlewood C. Misdiagnosis of the vegetative state: Retrospective study in a rehabilitation unit. British Medical Journal 1996;313:13-16.

5. Childs NL, Mercer WN, Childs HW. Accuracy of diagnosis of persistent vegetative state. Neurology 1993;43:1465-1467.

6. Giacino JT, Kalmar K, Whyte J. The JFK Coma Recovery ScaleRevised: Measurement characteristics and diagnostic utility. Archives of Physical Medicine \& Rehabilitation 2004;85: 2020-2029.

7. Bekinschtein TA, Coleman MR, Niklison 3rd J, Pickard JD, Manes FF. Can electromyography objectively detect voluntary movement in disorders of consciousness? Journal of Neurology, Neurosurgery \& Psychiatry 2008;79:826-828.

8. The Multi-Society Task Force on PVS. Medical aspects of the persistent vegetative state (1). The New England Journal of Medicine 1994;330:1499-1508.

9. von Wild K, Laureys ST, Gerstenbrand F, Dolce G, Onose G. The vegetative state-a syndrome in search of a name. Journal of Medicine \& Life 2012;5:3-15.

10. Laureys S, Schiff ND. Coma and consciousness: Paradigms (re)framed by neuroimaging. Neuroimage 2012;61:478-491.

11. Bruno MA, Majerus S, Boly M, Vanhaudenhuyse A, Schnakers C, Gosseries O, Boveroux P, Kirsch M, Demertzi A, Bernard C, et al. Functional neuroanatomy underlying the clinical subcategorization of minimally conscious state patients. Journal of Neurology 2012; 259:1087-1098.

12. Hugger S, Schindler HJ, Kordass B, Hugger A. Clinical relevance of surface EMG of the masticatory muscles. (Part 1): Resting activity, maximal and submaximal voluntary contraction, symmetry of EMG activity. International Journal of Computerized Dentistry 2012;15:297-314

13. Nöjd N, Hannula M, Narra N, Hyttinen J. Electrode position optimization for facial EMG measurements for human-computer interface. Methods of Information in Medicine 2008;47:192-197.

14. De Luca CJ, Gilmore LD, Kuznetsov M, Roy SH. Filtering the surface EMG signal: Movement artifact and baseline noise contamination. Journal of Biomechanics 2010;43:1573-1579.

15. Bagnato S, Boccagni C, Sant'angelo A, Prestandrea C, Romano MC, Galardi G. Neuromuscular involvement in vegetative and minimally conscious states following acute brain injury. Journal of the Peripheral Nervous System 2011;16:315-321.

16. Natale M, Mirone G, Rotondo M, Moraci A. Intrathecal baclofen therapy for severe spasticity: Analysis on a series of 112 consecutive patients and future prospectives. Clinical Neurology \& Neurosurgery 2012;114:321-325.

17. Thibaut A, Chatelle C, Ziegler E, Bruno MA, Laureys S, Gosseries O. Spasticity after stroke: Physiology, assessment, and treatment. Brain Injury 2013;27:1093-1105.

18. Owen AM, Coleman MR, Boly M, Davis MH, Laureys S, Pickard JD. Detecting awareness in the vegetative state. Science 2006;313:1402.

19. Monti MM, Vanhaudenhuyse A, Coleman MR, Boly M, Pickard JD, Tshibanda L, Owen AM, Laureys S. Willful modulation of brain activity in disorders of consciousness. New England Journal of Medicine 2010;362:579-589.

20. Schnakers C, Perrin F, Schabus M, Majerus S, Ledoux D, Damas P, Boly M, Vanhaudenhuyse A, Bruno MA, Moonen G, et al. Voluntary brain processing in disorders of consciousness. Neurology 2008;71:1614-1620.

21. Cruse D, Chennu S, Chatelle C, Bekinschtein TA, FernándezEspejo D, Pickard JD, Laureys S, Owen AM. Bedside detection of awareness in the vegetative state: A cohort study. Lancet 2011;378: 2088-2094.

22. Bruno MA, Vanhaudenhuyse A, Thibaut A, Moonen G, Laureys S. From unresponsive wakefulness to minimally conscious PLUS and functional locked-in syndromes: Recent advances in our understanding of disorders of consciousness. Journal of Neurology 2011; 258:1373-1384.

23. Bekinschtein TA, Manes FF, Villarreal M, Owen AM, Della-Maggiore V. Functional imaging reveals movement preparatory activity in the vegetative state. Frontiers in Human Neuroscience 2011;5:5.

24. Vanhaudenhuyse A, Boly M, Bruno MA, Gosseries O, Demertzi A, Kirsch M, Tshibanda JF, Thonnard M, Charland-Verville V, Laureys S, et al. 2012. Functional imaging and impaired consciousness. In: Schnakers C, Laureys S, editors. Coma and disorders of consciousness. London: Springer-Verlag. pp 25-34.

25. Leeb R, Sagha H, Chavarriaga R, Millán Jdel R. A hybrid braincomputer interface based on the fusion of electroencephalographic and electromyographic activities. Journal of Neural Engineering 2011;8:025011. 